\title{
ATLAS Public Website: Evolution to Drupal 8
}

\author{
Mariana Velho ${ }^{a, *}$ on behalf of the ATLAS Collaboration \\ ${ }^{a}$ European Laboratory for Particle Physics, CERN, \\ Espl. des Particules 1, Meyrin, Switzerland \\ E-mail: mariana.velho@cern.ch
}

Four years after the deployment of the ATLAS public website using the Drupal 7 content management system, the ATLAS Education \& Outreach group has completed its migration to the new CERN Drupal 8 infrastructure. We present lessons learned from the development, usage, and evolution of the original web site, and how the choice of technology helped to shape and reinforce our communication strategy. We then discuss tactics for the migration to Drupal 8, including our choice to use the CERN Override theme. This theme was developed by the CERN web team to support clients like the ATLAS experiment Collaboration to develop web sites in the relatively complex and nonintuitive environment of Drupal. Furthermore, CERN has encouraged usage of this theme to ease maintenance and future migration. We present the effects this choice has on the design, implementation, and operation of the new site.

The Ninth Annual Conference on Large Hadron Collider Physics - LHCP2021

7-12 June 2021

Online

*Speaker

(c) Copyright owned by the author(s) under the terms of the Creative Commons

Attribution-NonCommercial-NoDerivatives 4.0 International License (CC BY-NC-ND 4.0). 


\section{Introduction}

Communication of scientific goals, progress and achievements is a basic necessity of all fundamental research. This is particularly true for the large, publicly-funded international collaborations of the Large Hadron Collider (LHC) at CERN [1]. Not only does the public have the right to know how and towards what aim their resources are being spent, but they are key stakeholders of the knowledge that is being gained.

Beyond the social responsibility of scientific communication, however, lies its ability to reach world-wide audiences with messages that go above and beyond the reporting of results. These can include the tools of science and the methodology of the scientific process, the value of international collaboration, and the contributions being made to health, finance, security and other indirectly related fields. The ATLAS Collaboration takes advantage of its communication platforms to bring these messages to a variety of global audiences, thus securing support for the field through key stakeholders and decision makers, and sparking interest in the broader public and the next generation of scientists and engineers.

The primary platform supporting this communication is the ATLAS public website [2]. The ATLAS public website gives access to:

- Descriptions of the ATLAS experiment [3] and the collaboration.

- Multimedia educational resources for teachers, collaboration members, media and others.

- Updates (News, Briefings, Features, Portraits, Press Statements, Blogs).

- Other key communication platforms (social media, image \& video hosting sites, visits).

\section{Development and evolution of the existing website}

\subsection{History of the development}

The current ATLAS public website was designed in 2015 and released in March 2016, using version 7 of the Drupal content management system. The decision by CERN and many of its experiments and projects to turn to Drupal followed discussions dating back to 2010, with the creation of the ENTICE Task Force [4]. Priorities for many of the public CERN-based websites were determined to include reliable content management, well-developed open-source libraries, security, and integration with existing tools, such as the CERN Document Server (CDS) [5].

CERN underwent a redesign and implementation of its own internal and external websites over the next several years, then offered the experiments and other CERN-based projects Drupal infrastructure and technical support to follow their lead. After preliminary studies in 2014, the ATLAS website became one of the first large-scale sites to do so by re-developing its public appearance. A complete description of that development was presented at CHEP 2015 in Okinawa, Japan [6]. The site's general structure was based on analysis of data from surveys of the public and collaboration members using the existing public website, as well as interviews with key stakeholders planning to use and/or contribute to the new site. These included collaboration members active in communication and public engagement, scientists, and educators from outside the collaboration, members of the ATLAS management, and experts in web user experience. The resulting structure comprised three main pillars: descriptions of the experiment (Discover), access to relevant material (Resources) and current activities (Updates).

An external web firm [7] worked closely with a small team of collaboration members, using fast prototype iterations, to create the initial design. Following approval by the steering committee and ATLAS management, another external firm [8] completed implementation, delivering a Drupal 7 theme, complete with design elements and structure components built on the existing CERN core infrastructure. The content was developed by the ATLAS Education \& Outreach team 
and/or imported from existing websites.

\subsection{Evolution of content and design}

Several additions and adjustments of the design have been implemented by the ATLAS team since the public release. For example, short codes were extended to allow more flexibility in the design of Update pages. Short codes are a user-defined mark-up language designed to give instructions to the template code (PHP) and the style files (CSS) to control layout of the relevant nodes. They supplement functionality of the existing editing tools and are necessary, as Drupal 7 does not have a graphical front-end comparable to other content management systems, such as WordPress [9].

Adjustments were also made to improve the portability of content to various size screens. Much of this work was handled through the tuning of parameters in the style files, although some coding was needed in a few of the existing templates. New content types, such as Glossary Terms and Job Postings, were added, as were new views of content. This added the ability to browse all Updates or any one type of Update on a single page. New categories of Updates, which are implemented as taxonomies, were developed, including Features and Portraits. These allowed the team respectively to highlight longer in-depth articles on specific physics topics and to develop biographical articles on members of the collaboration.

Many of the style and functionality changes were made in response to user feedback, others to simplify and improve usage of the site by the content providers. Social media tabs [10] were implemented for sharing content and a feedback tab brought users to a form soliciting their input. Drupal infrastructure upgrades were handled by the CERN core web team, although several modules were added to the site for additional functionality, including image handling, Google analytics $[11]$ and PDF creation, for example.

\section{ATLAS migration process}

\subsection{Drupal Structure and Appearence}

The CERN Override theme provides tools to modify basic parameters, such as font, logo and colour palette, so the ATLAS design guidelines [12] could be applied to the new site. Other more detailed changes, such as the look and feel of the header, main navigation menu, search bar and footer, required significant changes to the CSS style files on the website's server. Careful checks need to be made on each page for all expected screen sizes to avoid unexpected effects. The need for these modifications drove the decision to adopt the CERN Override theme, as maintenance of a distinct visual identity is a necessary aspect of public communication.

The structure of a Drupal site comprises content types, views, blocks, and taxonomies, which allow for the development and structured presentation of the individual pages or nodes. These needed to be rebuilt from scratch in Drupal 8, but were primarily copies of the same components on the Drupal 7 site. Improvements were implemented, based on experience from usage of the existing site. In addition, the flexibility of the new Drupal 8 components allowed for a reduction of the number of content types and views, simplifying both development and maintenance. The Landing Page content type, for example, was used to replace several dedicated content types from the existing pages and allowed deployment of new layout components implemented by the CERN team.

\subsection{Large-Scale Migration}

Although landing pages, views and blocks can be translated by hand, there are a few thousand nodes of content that needed to be ported in a more automatic manner. These include Updates and Job Postings on the existing public site, and Virtual Visits previously hosted on a different Drupal 
7 site. In this case, the content fields were carefully mapped from old to new databases.

In order to preserve the existing Update layouts, a dedicated module was written to interpret embedded short codes in a manner similar to the current site. In addition, the Update content type includes a new field that allows for usage of the Bricks package [13], with the hope that this module will become mature enough one day to replace the short codes.

\section{Summary}

The ATLAS Education \& Outreach group has successfully and seamlessly migrated its public website from version 7 to version 8 of the Drupal content management system. The team built the new site using the CERN Override theme with significant support from the CERN web development team. The new pages are already well into use and new content is being added daily. Several new types of content structures for the hosting of educational resources have been developed and number of views of the website continues to increase.

Some of the more significant challenges that were faced included implementation of a look and feel that is distinct from CERN and based on the ATLAS visual identity, usage of a reduced number of generic content types to simplify maintenance, large-scale porting of a few thousand nodes of existing content, and development of a style that is better tuned for a large variety of screen sizes and use cases. The lack of an easy-to-use professional layout front-end, however, remains a challenge to be tackled for ATLAS and the international Drupal community. 


\section{References}

[1] The Large Hadron Collider: https://home.cern/science/accelerators/largehadron-collider.

[2] ATLAS Public Website: https://atlas.cern.

[3] ATLAS experiment detector description: JINST 3 (2008) S08003.

[4] ENTICE is an initiative to provide content management solutions for the CERN community: https://entice.web.cern.ch.

[5] CDS is the CERN Document Server: https://cds. cern.ch.

[6] S. Goldfarb, et al., J. Phys. Conf.: Ser. 664 (2015), 052015.

[7] Mark Boulton Design: https: / / www. markboultondesign.com.

[8] Vector Media Group: https: / / www . vectormediagroup.com.

[9] WordPress is an Open Source Content Management System:

https: / / wordpress.org.

[10] AddThis provides tools for sharing content to Social Media:

https://www.addthis.com.

[11] Google analysis views of website statistics: https: / / analytics.google.com.

[12] ATLAS design guidelines: http://atlas.cern/design.

[13] Bricks is a Drupal 8 layout module: https://www. drupal.org/project/bricks. 